\title{
Higgs Boson Decay to Light Jets at the LHC
}

\author{
Zhuoni Qian* \\ Center for Theoretical Physics of the Universe, Institute for Basic Science (IBS), Daejeon, \\ 34126, Korea \\ E-mail: zhuoniqianegmail.com
}

\section{Linda M. Carpenter}

Department of Physics, Ohio State University 191 West Woodruff Ave., Columbus OH, 43210, U.S.A.

E-mail: lmc@physics.osu.edu

\section{Tao Han}

Pittsburgh Particle Physics, Astrophysics, and Cosmology Center, Department of Physics and Astronomy, University of Pittsburgh, 3941 O'Hara St., Pittsburgh, PA 15260, USA

Department of Physics, Tsinghua University, P.R. China

Collaborative Innovation Center of Quantum Matter, Beijing, China

E-mail: than@pitt.edu

\section{Khalida Hendricks}

Department of Physics, Ohio State University 191 West Woodruff Ave., Columbus OH, 43210, U.S.A.

E-mail: hendricks.189@osu.edu

\section{Ning Zhou}

Department of Physics, Tsinghua University, P.R. China

Collaborative Innovation Center of Quantum Matter, Beijing, China

E-mail: nzhou2015etsinghua.edu.cn

We study the Higgs boson $(h)$ decay to two light jets at the 14 TeV High-Luminosity-LHC (HLLHC), where a light jet $(j)$ represents any non-flavor tagged jet from the observational point of view. The decay mode $h \rightarrow g g$ is chosen as the benchmark since it is the dominant channel in the Standard Model (SM), but the bound obtained is also applicable to the light quarks $(j=u, d, s)$. We estimate the achievable bounds on the decay branching fractions through the associated production $V h\left(V=W^{ \pm}, Z\right)$. Events of the Higgs boson decaying into heavy (tagged) or light (untagged) jets are correlatively analyzed. We find that with $3000 \mathrm{fb}^{-1}$ data at the HL-LHC, we should expect approximately $1 \sigma$ statistical significance on the SM $V h(g g)$ signal in this channel. This corresponds to a reachable upper bound $\mathrm{BR}(h \rightarrow j j) \leq 4 \mathrm{BR}^{S M}(h \rightarrow g g)$ at $95 \%$ confidence level. A consistency fit also leads to an upper bound $\mathrm{BR}(h \rightarrow c c)<15 \mathrm{BR}^{S M}(h \rightarrow c c)$ at $95 \%$ confidence level. The estimated bound may be further strengthened by adopting multiple variable analyses, or adding other production channels.

The 39th International Conference on High Energy Physics (ICHEP2018)

4-11 July, 2018

Seoul, Korea

${ }^{*}$ Speaker. 


\section{Signal and Background Processes}

While the precision measurements of those couplings will continue in the LHC experiments, it is imperative to seek other "rare decay" channels, such as the clean mode to $\mu^{+} \mu^{-}$[1]. For the other hadronic channels, it is extremely challenging at the LHC. The most promising channel is through $W(Z) h$ associated production, with $W / Z$ decaying leptonically to serve as effective triggers, and the Higgs signal detected from the construction of its hadronic decays. In the work we study Higgs decay to a pair of light un-tagged jets $h \rightarrow j j$, in the associated production channel.

Depending on the production mechanisms and the final states, we consider the following subprocesses

$$
\begin{aligned}
& q \bar{q} \rightarrow W^{ \pm} h \rightarrow \ell^{ \pm} v+j j, \\
& q \bar{q}, g g \rightarrow Z h \rightarrow\left\{\begin{array}{l}
\ell^{+} \ell^{-}+j j, \\
v \bar{v}+j j,
\end{array}\right.
\end{aligned}
$$

where $\ell=e, \mu$ and $j=g$ or $u, d, s$. Practically, $j$ is a gluon as expected in the SM. We thus generically denote the SM signal by $V h(g g)$, whenever convenient. For the SM backgrounds, we mainly consider the dominant irreducible background process $V+j j$ at LO, where the $V$ decays and contributes accordingly to the three signal channels.

\section{Signal Selection}

In further studying the signal characteristics in Eqs. (1.1) and (1.2), we categorize the channels according to the zero, one, or two charged leptons from the vector boson decays. In addition, the signal has two leading jets from the Higgs decay, and at high $p_{T(h)}$, the distance between the two hadronic jets can be estimated as $R_{j j} \approx \frac{1}{\sqrt{z(1-z)}} \frac{m_{h}}{p_{T(h)}}$, where $z, 1-z$ are the momentum fraction of the two jets. The LO parton-level distributions of three kinematic discriminants for the $Z h$ channel, the transverse momentum $p_{T(Z)}$, the jet separation $R_{j j}$, and the di-jet invariant mass $m_{j j}$, are studied to optimize the cuts.

To suppress the huge QCD di-jet backgrounds, we further optimize the reconstruction of the Higgs mass. Specifically we propose a modification of the two-jet-resolved method by including possible additional jets in the decay neighborhood - a "resolved Higgs-vicinity" method. After clustering the jets with anti-kt $\Delta R=0.4$, two leading $p_{T}$ jets within $R_{\max }$ are clustered as the "Higgs-candidate". Then additional jets $j^{\prime}$ are also clustered to the "Higgs candidate" in sequence of angular vicinity, whenever $R_{H j^{\prime}} \leq R_{\max }$. We choose $R_{\max }=1.4$.

\section{Alternative Discriminants with Missing Energies}

We note that a momentum balance discriminant could be efficient to select signal over background by itself. We define a discriminant by subtracting the scalar sum of the transverse momenta of the visible particles with the missing transverse energy in the event,

$$
T v Q \equiv \Sigma_{i}\left|p_{T i}\right|-\left|E_{T}^{\prime}\right| .
$$




\begin{tabular}{|c|c|c|c|c|}
\hline$\sigma(\mathrm{fb})$ & $\ell^{+} \ell^{-}+j j$ & $\ell^{ \pm}+E_{T}+j j$ & $E_{T}+j j$ & combined \\
\hline$V h$ signal & $7.0 \times 10^{-2}$ & $4.1 \times 10^{-1}$ & $3.6 \times 10^{-1}$ & \\
\hline$V j j$ background & $2.4 \times 10^{2}$ & $2.5 \times 10^{3}$ & $1.6 \times 10^{3}$ & \\
\hline $\mathscr{S}$ & 0.25 & 0.61 & 0.49 & 0.82 \\
\hline $\mathscr{S}_{\text {sys }}$ & 0.09 & 0.17 & 0.17 & 0.26 \\
\hline
\end{tabular}

Table 1: Signal significance achieved from each channel and combined results for both statistics and systematics dominance.

This is a version of a momentum balance discriminant, referred as $T v Q$ (Transverse event Quality). Another simple discriminant is the transverse angular variable $\phi_{Z h}$, defined as the angle between the missing transverse energy vector and the vector sum of the visible $p_{T}$. We examined the selective cuts $(-30 \mathrm{GeV}<T v Q<10 \mathrm{GeV})$ or $\left(\pi-0.5<\phi_{Z h}<\pi+0.5\right)$ and found them comparably effective in separating the signal from the backgrounds.

\section{Results and Discussion}

\subsection{Signal significance}

As shown in Table 1 , the pure statistical estimation gives a $0.82 \sigma$ significance. We consider sideband fitting to control the systematic uncertainties [2], and the significance follows as

$$
\mathscr{S}_{\text {sys }}=\frac{N_{\text {sig }}}{\varepsilon_{B} \times N_{\mathrm{bkg}}}
$$

where $\varepsilon_{B}$ is the fitted background percentage uncertainty. Further reduction of non-statistic uncertainties is expected with more dedicated background fitting schemes, once real data is available from experiments.

\subsection{Bounds on the branching fractions and correlations with $h \rightarrow b \bar{b}, c \bar{c}$}

The interpretation of these results to bound on individual Higgs decay channels needs further discussion. The signal we have been searching for in this study really is $h \rightarrow j^{\prime} j^{\prime}$ where $j^{\prime}$ is an "un-tagged jet" including possible $b, c$ and $j(g, u, d, s)$ contributions.

Assuming each category is statistically independent and following Gaussian statistics. We combine the three categories to get the three dimensional contour constraint on $\left\{\mu_{b}, \mu_{c}, \mu_{j}\right\}$ correlatively based on the relation

$$
\begin{aligned}
\mathscr{S}^{2} & >\sum_{a} \chi_{a}^{2}=\sum \frac{\left(x_{a}-\bar{x}_{a}\right)^{2}}{\sigma_{a}^{2}}=\sum_{a} \frac{\left(\sum_{i} \varepsilon_{a i}^{2} \mathrm{BR}_{i} N_{\mathrm{sig}}^{\text {prod }}-\sum_{i} \varepsilon_{a i}^{2} \mathrm{BR}_{i}^{\mathrm{SM}} N_{\mathrm{sig}}^{\text {prod }}\right)^{2}}{\left(\sqrt{N_{\mathrm{bkg}}}\right)^{2}} \\
& =\sum_{a} \frac{\left(\sum_{i} e_{a i} \mu_{i}-1\right)^{2}}{\left(1 / \mathscr{S}_{a}\right)^{2}}
\end{aligned}
$$

where $\mathscr{S}_{a}$ is the significance from each category measured by experiments, and $e_{a i}$ are the efficiencies from each decay channel $i$ in category $a$ taken from tagging prospects. The correlated signal 


\begin{tabular}{|c|c|c|c|}
\hline $\mathscr{L}\left(\mathrm{fb}^{-1}\right)$ & $\bar{\kappa}_{u}\left(\kappa_{u}\right)$ & $\bar{\kappa}_{d}\left(\kappa_{d}\right)$ & $\bar{\kappa}_{s}\left(\kappa_{s}\right)$ \\
\hline $300\left(\right.$ un-tagged $\left.j^{\prime} j^{\prime}\right)$ & $1.2(2600)$ & $1.2(1200)$ & $1.2(61)$ \\
\hline 3000 (un-tagged $\left.j^{\prime} j^{\prime}\right)$ & $0.65(1500)$ & $0.65(680)$ & $0.65(34)$ \\
\hline Current Global Fits [3] & $0.98(2200)$ & $0.97(1000)$ & $0.70(37)$ \\
\hline
\end{tabular}

Table 2: Extrapolated upper bounds at $95 \% \mathrm{CL}$ on the light-quark Yukawa couplings $\bar{\kappa}_{q}=y_{q} / y_{b}^{\mathrm{SM}}\left(\kappa_{q}=\right.$ $\left.y_{q} / y_{q}^{\mathrm{SM}}\right)$ for $q=u, d, s$.

strengths are constrained in a 3-dimensional contour of $\left(\mu_{b}, \mu_{c}, \mu_{j}\right)$, analyzed with statistical error (including systematical error). Sensitivities at $2 \sigma$ level can be obtained with $3000 \mathrm{fb}^{-1}$ data as

$$
\begin{aligned}
& \mathrm{BR}(h \rightarrow j j) \leq 4(9) \times \mathrm{BR}^{S M}(h \rightarrow g g), \\
& \mathrm{BR}(h \rightarrow c \bar{c})<15 \times \mathrm{BR}^{S M}(h \rightarrow c \bar{c}) .
\end{aligned}
$$

Although this bound on the $h \rightarrow g g$ channel is not nearly as strong as that from the production fit $g g \rightarrow h$ assuming the SM value, our study and results lay out the an independent test and the search for the direct decay of the Higgs boson to gluons and the light quarks.

\subsection{Bounds on light-quark Yukawa couplings}

As explained earlier, the bound on $h \rightarrow j j$ in Eq. (4.3) can be translated into those for the light quark Yukawa couplings. Assuming the SM $g g h$ coupling, and varying one light quark Yukawa $y_{q}$ at a time, we translate our bound on $\mu_{j}$ to the Yukawa couplings for light quarks $u, d, s$ by scaling the branching fraction with $\mu_{q} \propto y_{q}^{2}$. Our results are shown in Table 2. The upper bounds from our study of Higgs decay to light jets are comparable to those derived from the Higgs production kinematics, and provide complementary information to existing approaches.

\section{Acknowledgments}

The work of T.H. and Z.Q. was supported in part by the U.S. Department of Energy under grant No. DE-FG02-95ER40896, in part by PITT PACC. Z.Q. was also supported in part by a PITT PACC Predoctoral Fellowship from School of Art and Science at University of Pittsburgh. The work of K.H. was supported by the National Science Foundation Graduate Research Fellowship under Grant No. DGE-134012. The work of L.C. and K.H. was supported by the U.S. Department of Energy under grant No. DE-SC0013529.

\section{References}

[1] T. Han and B. McElrath, Phys. Lett. B 528, 81 (2002) doi:10.1016/S0370-2693(02)01208-X [hep-ph/0201023].

[2] L. M. Carpenter, T. Han, K. Hendricks, Z. Qian and N. Zhou, Phys. Rev. D 95, no. 5, 053003 (2017) doi:10.1103/PhysRevD.95.053003 [arXiv:1611.05463 [hep-ph]].

[3] A. L. Kagan, G. Perez, F. Petriello, Y. Soreq, S. Stoynev and J. Zupan, Phys. Rev. Lett. 114, no. 10, 101802 (2015) doi:10.1103/PhysRevLett.114.101802 [arXiv:1406.1722 [hep-ph]]. 